\title{
Research Note \\ Stellar photometry with the Carte du Ciel plates
}

\author{
I. Method ${ }^{\star}$ \\ F. Lamareille ${ }^{1,2}$, J. Thiévin ${ }^{1,3}$, B. Fournis ${ }^{1,3}$, P. Grimault ${ }^{1,3}$, L. Broquet ${ }^{1,4}$, and E. Davoust ${ }^{1}$ \\ 1 UMR 5572, Observatoire Midi-Pyrénées, 14 avenue E. Belin, 31400 Toulouse, France \\ 2 UFR PCA, Université Paul-Sabatier, 118 route de Narbonne, 31400 Toulouse, France \\ 3 UFR SPM, Université de Rennes 1, Campus de Beaulieu, 35000 Rennes, France \\ ${ }^{4}$ Université de Cergy-Pontoise, 33 boulevard du Port, 95011 Cergy-Pontoise Cedex
}

Received 19 November 2002 / Accepted 19 February 2003

\begin{abstract}
We present a method for measuring the magnitude of stars on the photographic plates of the Carte du Ciel. There are between 95 and 162 non-variable stars of known $B$ and $V$ magnitudes on each plate, which covers an area of 2 square degrees on the sky. The densities of these stars, measured with a commercial scanner, are used to calibrate the density-to-intensity conversion relation of each plate. The derived magnitudes decrease linearly away from the center and then brighten again more steeply toward the edge, presumably because of vigneting and coma. A distance- and density-dependent correction for these two effects is determined for each plate; this is necessary if one wants to determine the light curve of a given star with plates belonging to adjacent fields. We find a magnitude-dependent color effect, which is not understood, and thus not corrected for. The final accuracy of the calibration depends on the distance from the center of the plate, and is optimal near the center. The method is tested on five known variable stars.
\end{abstract}

Key words. methods: data analysis - techniques: photometric - stars: variables: general

\section{Introduction}

Toulouse Observatory was one of the four French Observatories to take part in the Carte du Ciel project (Debarbat 1988), and over 9000 photographic plates were taken between 1891 and 1966. The Toulouse zone is between $+4^{\circ}$ and $+11^{\circ}$ in declination. The plates were recently inventoried, restored and put in chemically neutral envelopes. The complete inventory is available on-line at webast . ast.obs-mip.fr/patrimoine/cdc_e.html

There are over one hundred fields of the Toulouse zone with more than ten plates, and one field has been photographed 41 times. Some of the plates only have short exposures $(0.3,2.5$ and $5 \mathrm{~min}$ ), which reduces the magnitude range for some fields (or the number of plates with fainter stars), and the sampling is never very uniform in time over the 75 years. Another problem arises from the grid, which is a series of lines printed on each plate, with a spacing of 5 arcmin, for facilitating the reduction at the time of the survey; stars that fall on that grid cannot be

Send offprint requests to: E. Davoust, e-mail: davoust@ast.obs-mip.fr

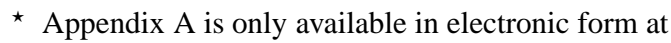
http://www.edpsciences.org accurately measured. On the other hand, since successive declination strips overlap by $1^{\circ}$ (there are even plates taken at $4.5^{\circ}$, and $10.5^{\circ}$ of declination), plates from adjacent fields overlap by at least a quarter of a field, thus some stars are visible on plates corresponding to two (and sometimes three) different adjacent fields.

While recent reanalysis of the astrographic catalogue or new reductions of the Carte du Ciel plates focused on astrometric applications (e.g. Urban et al. 1998; Ortiz-Gil et al. 1998), we chose to exploit the photometric resources of these plates, and, in particular, to search for new long-period variable stars. This first paper deals with the adopted reduction method and the first results on one field.

\section{The test sample of plates}

The field selected for this study is the most sampled one of the Toulouse zone, with 41 plates taken over the years. We did not specifically look for a field with known variable stars, because the long-period Mira variables are generally not very regular in period or in amplitude, and shorter-period variables would not be well sampled by our plates which are generally widely spaced in time. Since there is an overlap of a quarter of a plate 
Table 1. Test sample of plates.

\begin{tabular}{rclrr}
\hline \hline field & RA & Dec & $N$ plates & $N$ stars \\
\hline 0 & $17 \mathrm{~h} 56 \mathrm{~m}$ & 5 & 29 & 272 \\
1 & $17 \mathrm{~h} 52 \mathrm{~m}$ & 6 & 5 & 156 \\
2 & $17 \mathrm{~h} 52 \mathrm{~m}$ & 4 & 9 & 162 \\
3 & $18 \mathrm{~h} 00 \mathrm{~m}$ & 4 & 5 & 207 \\
4 & $18 \mathrm{~h} \mathrm{00m}$ & 4.5 & 5 & 200 \\
5 & $18 \mathrm{~h} \mathrm{00m}$ & 6 & 7 & 184 \\
\hline
\end{tabular}

between plates of different declinations, and that an additional series of plates at $4.5^{\circ}$ were taken, we also used the plates of fields at adjacent declinations.

The SIMBAD database of Centre de Données Astronomique de Strasbourg (simbad.u-strasbg.fr/ simbad) was queried for stars in the area of the sky covered by the plates, brighter than $B=12$, and which were included in one of the four catalogues SAO, HIP, HD, GSC, (which stand for Smithsonian Astrophysical Observatory, Hipparcos, Henry Draper and Guide Star Catalogue, respectively). Variable stars were excluded from the query. The data requested from SIMBAD were the star name in one of the four catalogues, the equatorial coordinates (epoch: year of the plate; equinox: 2000). The $B$ and $V$ magnitudes were provided by the Tycho catalogue, available through VIZIER at the same data center.

The selected sample of plates is described in Table 1, which gives the coordinates, number of measured plates, and total number of catalogued stars in each field. The central field is numbered 0 , and the adjacent fields are arbitrarily numbered from 1 to 5. There are fewer measured plates than available for a given field, because we did not use the short-exposure ones (less than $10 \mathrm{~min}$ ). The actual number of stars measured on a plate depends on the exposure time (which varies between 10 and $60 \mathrm{~min}$ ) and on the location of the star on the plate (stars that fell on the grid had to be discarded). There was a total of 60 plates, and the total number of measured stars was 8014 . In studying the color effect (Sect. 6), we averaged all the measures of a given star, regardless of the field, and there were a total of 565 points. In studying the distance effect (Sect. 5), we took averages over each field separately and there were a total of 1020 measures, since in this case a star could be in one, two or at most three fields.

\section{Digitizing photographic plates}

The plate densitometer scanner (PDS) has been the standard tool for decades of photometric work on photographic plates. However, the advent of inexpensive scanners prompted us to examine the feasability of using a commercial scanner for our project. We acquired an AGFA SNAPSCAN 1236S, which is adapted for transparent documents, and provides a maximum optical resolution of $600 \times 1200 \mathrm{dpi}$. One of us (L.B.) tested it with a square resolution of $600 \mathrm{dpi}$. He found that the densities of a set of sensitometric spots on a plate remain constant, provided the plate is placed in the center of the scanning area. This scanner was thus adopted for digitization at 600 dpi, which corresponds to a total size of about $3600 \times 3600$ pixels, and a pixel size of 2.5 arcsec, an acceptable resolution considering the instrumental resolution of the telescope and local seeing.

The dynamics of the scanner is not that of a PDS, the measured densities range from 0 (black) to 255 (transparent). We optimized the dynamic range of each plate by examining the histogram of densities and setting the minimum density at the level of the brightest pixel and the maximum density of 255 slightly beyond the peak which corresponds to the sky background. A second peak beyond that value corresponds to the unexposed edge of the plate. Note that these densities vary in opposite sense to the commonly accepted definition.

The resulting TIFF files were then converted to FITS format and analyzed with the ESO-MIDAS image treatment software.

\section{Measuring the plates}

The first step in this analysis is to determine the exact scale and orientation of each plate, in order to identify the stars on the plate with the catalogued stars. Four reference stars are selected by visual inspection of the plate image, one near the center of the plate and three in the outer parts. Their equatorial and pixel coordinates are used to determine the relation between the two reference frames.

The conversion of equatorial coordinates on the sky to rectangular coordinates on the plate is done with the equations of gnomonic projection (see, e.g., Eichhorn 1974, Eq. (2.9)). The pixel coordinates are then obtained by scaling and rotation. The scaling factor between the rectangular and pixel coordinates is determined by comparing the distances between the four reference stars in the two reference frames, and the angle between the two reference frames is the average of the values for the four reference stars. Once these two parameters are determined, the equatorial coordinates of all the other stars are converted to pixel coordinates.

The next step in the analysis is to determine the magnitudes of the stars. There are two methods for doing this on photographic plates, in the absence of sensitometric spots. The first one relies on the diameter of the stars as a measure of their magnitude. It has the advantage of not being affected by the saturation of the image of bright stars, especially on copies of plates. This is the method used for the National Geographic - Palomar Observatory Sky Survey (King \& Raff 1977; Humphreys et al. 1991), the ESO/SRC Sky Atlas (King et al. 1981; Pierre 1986), the Second Cape Photographic Catalogue (de Vegt et al. 1993), among others (for a review of older studies, see Hearnshaw 1996).

The second method is to measure the densities of stars of know magnitude and to derive a density to intensity conversion law. It has been used for the Twin Astrographic Catalog of the US Naval Observatory (Zacharias \& Zacharias 1999), the Schmidt plates of CERGA (Mohan \& Crézé 1987), the plates of Sonneberg Observatory (Kroll \& Neugebauer 1993), the Carte du Ciel plates (Ortiz-Gil et al. 1998). Most of them fit a twodimensional Gaussian to the image, which enables corrections for saturation effects.

We adopted the second method, without a Gaussian fit. The densities were measured inside a rectangular aperture including 
the star and the corresponding sky background was measured in a similar aperture near the star. It would have been better to map and eliminate the large-scale variations of the sky background over the plates than to measure the sky background locally, but the grid printed on the plates prevented us from doing this.

Many plates have two or three exposures. We usually measured the two or three stellar densities separately, but used the same sky for all. Bright stars with overlapping images were measured in a single aperture. The total stellar density was simply determined by subtracting the sky density from the rough stellar density.

In practice, after a plate image is selected, the locations of the four calibrating stars are pointed on the screen; the scale and orientation of the plate are determined, and the pixel coordinates of all the other stars are calculated. The first star is shown on the screen and a cross is plotted at the assumed position of the star. The actual position may occasionally be somewhat off the predicted one, when no proper motion information is available for that star. The user measures the densities in apertures on and around the stellar image. The software then moves to the next star. The measurements are automatically stored in a file, one per plate.

In the case of plates with multiple exposures, two options are possible for the next step. One can either consider the total stellar density as the sum of the two or three individual stellar densities, or consider the individual stellar densities as resulting from stars of different magnitudes, the magnitude difference being $-2.5 \log \left(t_{2} / t_{1}\right)$, where $t_{1}$ and $t_{2}$ are the exposure times. The second option is only useful if one needs a calibration curve that extends beyond the faintest standard stars available for the given plate. Its main drawback is that the different exposure times given in the logbook are not very precise. If one uses the nominal exposure times, one finds systematic offsets between the two or three sets of data points. One then has to determine the optimal exposure time ratios by shifting the sets of data points until the dispersion reaches a minimum. The derived exposure times may be off by up to $50 \%$ with respect to the nominal ones, which is worrysome. Another drawback of this second option is that images of bright stars overlap when the shift between exposures is small; estimating the contribution of each exposure to their density then becomes difficult if not impossible. The drawback of the first option is that the third image of some faint stars is sometimes below the sensitivity threshold of the emulsion. We adopted the first option, but used the sum of the densities of the two longest exposures in the case of triple exposures.

We normalize the measured densities by that of the brightest star on the plate:

$D_{i}=\frac{d_{i}-d_{\text {isky }}}{d_{m}-d_{\text {msky }}}$

where $d_{i}$ and $d_{m}$ are the measured density of star $i$ and of the brightest star respectively, while $d_{\text {isky }}$ and $d_{\text {msky }}$ are the sky densities near these stars. In the final calibration, we in fact normalized all densities by the largest measured density among all 60 plates, in order to be able to study opacitance-dependent effects over all plates.

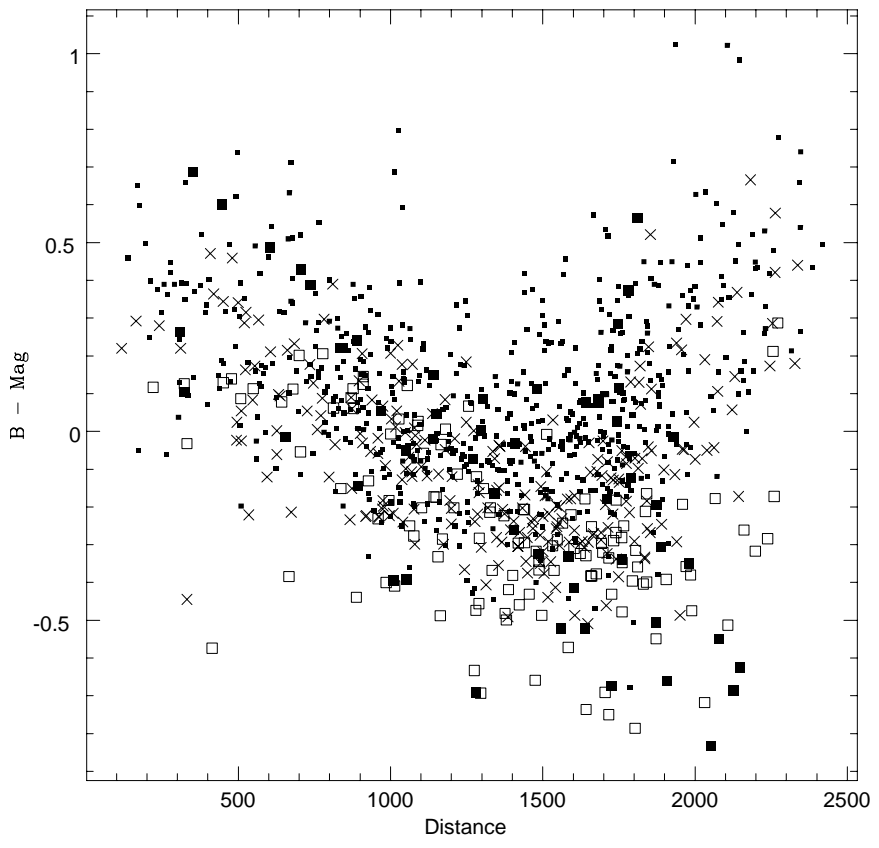

Fig. 1. Mean difference between $B$ and measured magnitude (Mag) as a function of distance from the center of the plate (in pixel units), for each star of a given field. Full squares correspond to stars with $B<9$, open squares to $9 \leq B<10$, crosses to $10 \leq B<11$, and dots to $B \geq$ 11. Beyond 1300 pixels, the dependence on distance is not the same for bright and faint stars.

In order to linearize as much as possible the response of the emulsion to the incoming flux, we convert the normalized densities $D$ to opacitances $\omega$ such that:

$\omega=10^{D}-1$.

The relation between $\log (\omega)$ and $B$ magnitude adopted as provisional calibration law for use in the next section is a polynomial of order 2 in $\log (\omega)$. It is preferred to the usual linear law because it compensates better for the saturation effect of bright stars (Zacharias \& Zacharias 1999).

\section{Correction for vigneting and for coma}

A total of 8014 densities were measured on 60 plates and converted to magnitudes using this preliminary conversion law. A detailed study of the difference $(B-\mathrm{Mag})$ between nominal and derived magnitude of each star on different plates of the same field revealed a distance-dependent systematic effect. Figure 1 shows the mean difference as a function of distance from the center of the plate for each star of a given field: near the center and the edges of the plate, the derived magnitudes are larger than expected. Near the edge of the plates, the magnitude difference at a given distance depends on magnitude.

We have verified that the large positive deviations are indeed symmetrically distributed around the center and near the edges, and that the the smaller and negative differences $(B-$ Mag < 0.2$)$ are uniformly spread over the plate area. This central symmetry excludes an artefact of the scanner, and the systematic effect must be attributed to two competing effects, vigneting which attenuates the stellar brigthness outward, and, 
beyond a distance of about $1^{\circ}$ from the center, a brightening due to optical aberration in the lense which spreads the stellar image and thus modifies the total measured density of the star. The latter effect has been studied by Seares (1914), who finds a dependence on the magnitude of the star. We do find that most bright star are not affected to this outward apparent brightening. Turner (1902), using starcounts, showed that the number of stars per unit surface on the Carte du ciel plates of the Toulouse zone decreases outward beyond about 30 arcmin; he explains this effect by a curvatury of the focal plane.

The adopted correction for the effect is a second order polynomial in the distance $D$, to which is added an opacitancedependent term, to correct for the magnitude dependence of the effect.

The final calibration curve is then determined by non-linear multivariate least-squares fit of the following function:

$$
\begin{aligned}
\operatorname{Mag}=a_{1} & +\log (\omega)\left[a_{2}+a_{3} \log (\omega)\right] \\
& +D\left[a_{4}+D\left(a_{5}+a_{6} D\right)\right] \\
& +a_{7} \log (\omega)[1200-D]
\end{aligned}
$$

where the coefficients $a_{i}$ are the unknowns, $\omega$ is the opacitance and $D$ the distance (in pixels). For this purpose we used the subroutine $l$ fit (Press et al. 1992), after modifying it in the way indicated by the authors for multivariate regression.

\section{Color correction}

The brand of plates used for the Carte du Ciel was not recorded in the logbook, and their spectral response is unknown to us. Photographic plates are generally sensitive to the blue part of the spectrum, but a small color correction is expected between the photographic and $B$ magnitudes. Mohan \& Crézé (1987) find a correction of $0.162(B-V)+0.048(U-B)$ for IIa0 plates with the GG385 filter. Humphreys et al. (1991; their Fig. 1b) also find a positive slope in $(B-V)$ for blue $(103 \mathrm{a} 0)$ plates. The color correction derived from the data of Kroll \& Neugebauer (1993) on Sonneberg plates are of the order of $-0.17(B-V)$. These different values give us an idea of the order of magnitude to be expected for the color effect.

We were very puzzled to find that the color effect on our plates depends not only on $(B-V)$, but also on magnitude. This is shown in Fig. 2, where the color dependence of $(B-$ $\mathrm{Mag}$ ) is plotted for each star in two magnitude ranges, and a linear least-squares fit is plotted for three magnitude ranges. The mean slope for stars with $B<10$ is -0.36 . This effect seems to be important beyond $(B-V)=0.8$ only. Since there is no reason why the color correction should be magnitude-dependent, we believe that it is a side-effect of some other, unidentified, effect. We thus decided not to correct for it. The derived magnitudes are thus nominally in the $B$ band, but one should keep in mind that these magnitudes suffer from a systematic (unknown) color-dependent offset from the true $B$ magnitudes.

\section{Uncertainties on the derived magnitudes}

The main problem with our calibration method is that stars that are not reported as variable are not necessarily of

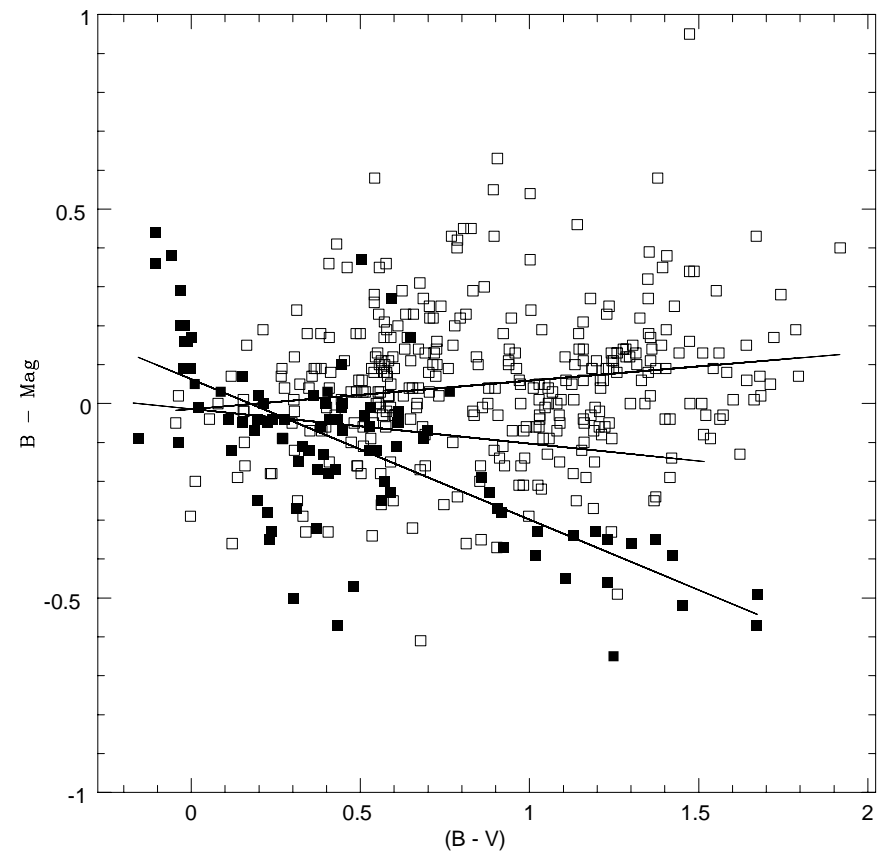

Fig. 2. Mean difference between $B$ and Mag, where Mag is the measured magnitude, as a function of color $(B-V)$. The straight lines of decreasing slope are linear correlations for $B<10$ (full squares), $10 \leq$ $B<11$ (data not shown, for the sake of clarity) and $B \geq 11$ (open squares).

constant magnitude. If we sort the 565 calibration stars by increasing scatter about their mean magnitude, this scatter increases monotonously, without any discontinuity to indicate at what level the variable stars show up. Thus we are never sure that the scatter about the mean derived magnitude of a given star is a measure of the uncertainty of our method or of variability of the star.

The sky background and stellar density measurements, which were both done by hand, 8014 times, are an important source of error. We estimate that the internal uncertainty on an individual measurement is about 0.08 mag, which is the standard deviation of magnitude determinations of the same stars by two different observers. In order to make up in part for this error, we decided to eliminate the worst magnitude (the one that departed most from the mean) for each star, assuming that it is an aberrant point.

Another source of error is the distance-dependent conversion law that we use, which is estimated from mean values of the measurements. This error can be considerably reduced by only using measurements from fields where the star is at about the same distance from the center. The cost is that there are fewer data points per star.

The fact that we do not make any color correction should have no effect, since this correction is in principle the same for all measurements of a given star. In practice, the resulting differences between nominal and measured magnitude increase the uncertainty on the coefficients of the conversion law.

There is another effect that we have not taken into account either, namely local variations of the plate sensitivity. Such an effect, which is considered for the Palomar Schmidt 


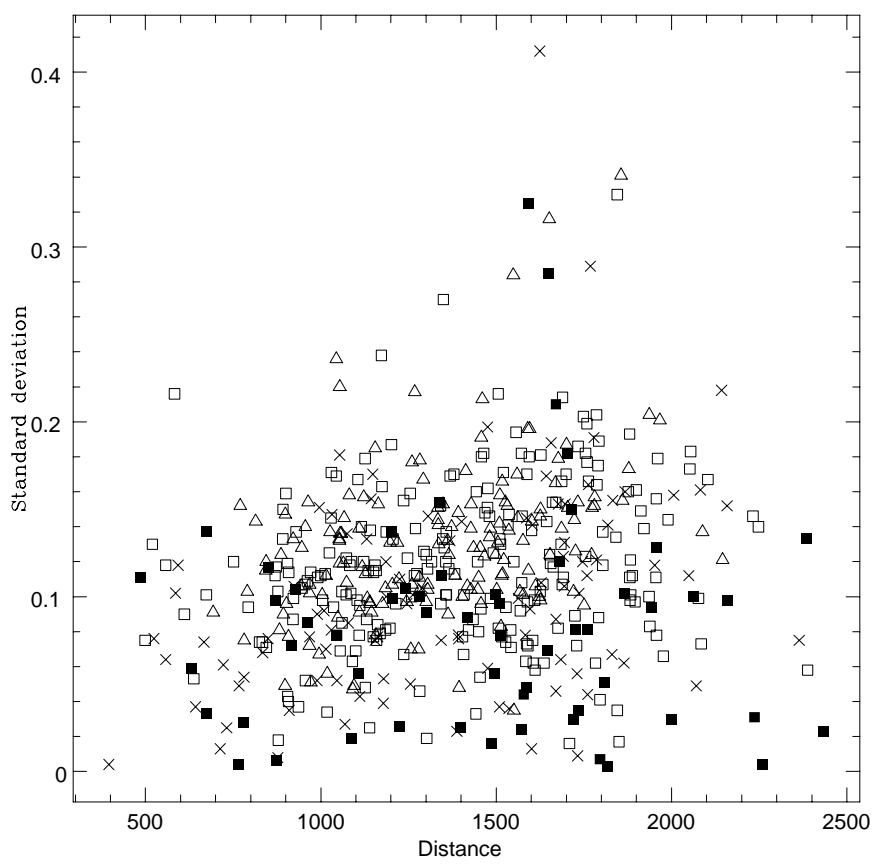

Fig. 3. Mean dispersion in magnitude over all plates (thus all six fields) for each star, as a function of mean distance from the center of the plate (in pixels). Different symbols show the scatter $\left(\sigma_{D}\right.$, in pixels) around the mean distance: $\sigma_{D}<60$ as full squares, $60 \leq \sigma_{D}<100$ as open squares, $100 \leq \sigma_{D}<300$ as crosses, and $\sigma_{D} \geq 300$ as open triangles. The stars which are at the same distance from the center on all plates (thus a small $\sigma_{D}$ ) have the most accurately determined magnitudes.

plates (Bucciarelli 1999), might be detectable if we had a large number of measured stars on the plate. We did try to find correlations between the sky background and the mean difference ( $B-$ Mag), but to no avail.

We have studied the accuracy of our magnitude determinations by computing various statistical parameters. We believe that the standard deviation of all derived magnitudes for a given star for all six adjacent fields is a good measure of the accuracy of our method. This is shown in Fig. 3, where the dispersion increases from about $0.12 \mathrm{mag}$ near the center to 0.22 in the outer regions, with a few stars above that limit. These stars are not necessarily variable, but rather stars for which the distance corrections were not adequate. On that figure, we distinguish stars with small distance differences from plate to plate, which generally have the most accurate magnitudes.

The overall accuracy is not as good (except near the center of the plates) as what has been achieved by Ortiz-Gil et al. (1998), who quote an accuracy of $0.1 \mathrm{mag}$, using a different, more elaborate, method. We have also computed the maximum magnitude range for each star, which shows a similar trend.

The only star that is definitely variable among the 565 calibration stars is SAO 123099. It has a very large scatter in magnitude, whether one considers field 0 only, or also the neighboring fields. Two other stars, SAO 122981 and SAO 123168 also have suspiciously large magnitude variations.

In order to check the validity of our reduction method and its accuracy, we have measured five known variable stars on the series of plates. Their variability study is in Appendix A.

\section{Conclusion}

We have calculated the photographic magnitude of stars on the Carte du Ciel plates. They are derived from the total density of the stars on the plates, converted to magnitudes with a transformation equation whose parameters are calibrated using between 95 and 162 stars of known magnitude on the plate. The magnitudes are accurate to about 0.2 to 0.4 mag, depending on the location of the star on the plate. We have measured the magnitudes of five variable stars, and found that their variations are compatible with the information available in the literature, except that the Mira variable V* v389 Oph has a smaller amplitude of variation than predicted in the period 1916-1960.

While the present method is satisfactory for detecting large amplitude variables, it may be useful to improve its accuracy. This can be done by adjusting a two-dimensional Gaussian to the stellar images. Overlapping images of bright stars will have to be treated by an iterative method, such as the one we designed to disentangle interacting elliptical galaxies (Prugniel et al. 1989). This approach of model spheroids with varying ellipticity and position angles should take care of the more complicated stellar images distorted by coma in the outer regions of the plates. It would also enable us to make a better correction for the coma effect, by designing a correction linked to the dispersion of the Gaussian adjusted to the stellar image, which should be a constant for a given plate.

Acknowledgements. We thank Elisabeth Griffin for valuable discussions and encouragements and Alain Fresneau for constructive criticisms of this paper. The internal reports (in French) on which this paper is based are available at webast.ast.obs-mip.fr/ patrimoine/cdc.html. Appendix A is published in electronic form only.

\section{References}

Bucciarelli, B. 1999, Acta Historica Astronomiae, 6, 45

Debarbat, S. (ed.), IAU Symp., 133

de Vegt, C., Murray, C. A., Zacharias, N., et al. 1993, A\&AS, 97, 985

Eichhorn, H. 1974, Astronomy of star positions (New York: Frederick Ungar Publishing Co.)

Hearnshaw, J. B. 1969, The measurement of starlight, two centuries of astronomical photometry (Cambridge University Press), 143

Hoffmeister, C. 1943, Kleinere Veröff. Berlin-Babelsberg, 28

Hoffmeister, C. 1967, AN, 290, 43

Humphreys, R. M., Landau, R., Ghigo, F. D., et al. 1991, AJ, 102, 395

Kholopov, P. N., Samus, N. N., Frolov, M. S., et al. 1998, Combined General Catalogue of Variable Stars 4.1 Ed (SIMBAD catalogue II/214A)

King, I. R., \& Raff, M. I. 1977, PASP, 89, 120

King, D. J., Birch, C. I., Johnson, C., et al. 1981, PASP, 93, 385

Kroll, P., \& Neugebauer, P. 1993, A\&A, 273, 341

Mohan, V., \& Crézé, M. 1987, A\&AS, 68, 529

Ortiz-Gil, A., Hiesgen, M., \& Brosche, P. 1998, A\&AS, 128, 621

Pierre, M. 1986, A\&A, 161, 408

Prugniel, Ph., Davoust, E., \& Nieto, J.-L. 1989, A\&A, 222, 5

Press, W. H., Teucholsky, S. A., Vetterling, W. T., \& Flannery, B. P. 1992, Numerical Recipes: The Art of Scientific Computing, 2nd ed. (Cambridge University Press)

Seares, F. H. 1914, ApJ, 39, 307

Turner, H. H. 1902, MNRAS, 62, 434

Urban, S. E., Corbin, T. E., Wycoff, G. L., et al. 1998, AJ, 115, 1212

Zacharias, N., \& Zacharias, M. I. 1999, AJ, 118, 2503 